\title{
Coverage and Rate Analysis for Limited Information Cell Association in Stochastic-Layout Cellular Networks
}

\author{
Prasanna Herath, Student Member, IEEE, Witold A. Krzymien, Senior Member, IEEE, \\ and Chintha Tellambura, Fellow, IEEE
}

\begin{abstract}
The complexity and uncertainty inherent in large cellular networks make the acquisition of location and channel information of all but perhaps a few neighbouring network nodes (base stations) difficult for a given user. Therefore, a cell association policy must operate with sparse information. Thus, the serving base station (BS) is proposed to be the one that provides the highest instantaneous signal-to-interference ratio (SIR) from among all BSs providing average received signal power exceeding a predetermined minimum. This policy is evaluated for the downlink of single-tier (homogeneous) and two-tier (heterogeneous) networks, and for the latter the key advantage of the proposed policy is its capability to enable traffic off-loading. Two methods to determine the minimum average signal power are given. Coverage probabilities and average rates of MSs in coverage are derived accounting for path loss, multipath fading and random locations of BSs in each tier. Analysis is verified by Monte-Carlo simulations. We observe that the instantaneous SIR and average received power of a few BSs are sufficient to achieve the coverage corresponding to the highest-SIR association, which in general requires instantaneous SIR information of a larger subset of a network. We also observe that in a two-tier network, the effect of strong interference from high power BSs, such as macro-BSs, can be limited by proper choice of minimum average received power for low power BSs.
\end{abstract}

Index Terms-Heterogeneous cellular networks, cell association, downlink transmission, stochastic geometry, point process theory, coverage probability, average data rate.

Manuscript received September 30, 2014; revised March 24, 2015, and July 31, 2015; accepted September 2, 2015. Date of publication N/A; date of current version N/A. This work was supported in part by Telecommunications Research Laboratories, the Rohit Sharma Professorship, TELUS, Mitacs, and the Natural Sciences and Engineering Research Council (NSERC) of Canada. Some of this work was presented at the 80th IEEE Vehicular Technology Conference (VTC 2014-Fall), Vancouver, Canada, Sepember 2014. The editor coordinating the review of this paper and approving it for publication was Dr. Chau Yuen.

Copyright (c) 2015 IEEE. Personal use of this material is permitted. However, permission to use this material for any other purposes must be obtained from the IEEE by sending a request to pubspermissions@ieee.org.

The authors are with the Department of Electrical and Computer Engineering, University of Alberta, Edmonton, Canada, T6G 1H9 (e-mail: prasanna@ualberta.ca, wak@ece.ualberta.ca, chintha@ece.ualberta.ca). P. Herath and W. A. Krzymień are also with the Telecommunications Research Laboratories, Edmonton, Canada, T5K 2M5.

Color versions of one or more of the figures in this paper are available online at http://ieeexplore.ieee.org. Digital Object Identifier 10.1109/TVT.201X.XXXXXXX

\section{INTRODUCTION}

Cellular networks must increase their capacity to satisfy exploding demand for data services. In 2014 the number of mobile-connected devices exceeded the world population, while 1.5 mobile devices per capita is projected by 2019 [1]. Over half of all these devices will be "smart" devices by 2019 creating a tremendous demand of over 24 Exabytes of information exchange per month [1]. To cater to these data-hungry smart devices, the number of base stations (BSs) increases rapidly each year, overwhelmingly by virtue of small BSs (pico and femto) being added to the existing network [2]. This evolution towards heterogeneity is making cellular networks more complex and irregular compared to the traditional carefully planned single-tier architecture.

In heterogeneous networks, the cell association policy plays an important role in providing the best userperceived rate [2]. Due to the uncertainty of locations of network nodes and users ${ }^{1}$, many association policies have been studied under different spatial models for single-tier (homogeneous) and multi-tier (heterogeneous) networks; see [3]-[13], and references therein. They can be categories as closest-BS [4]-[6], highest-signal-to-interferenceplus-noise ratio (SINR) (equivalently, highest-signal-tointerference ratio (SIR) in interference-limited networks) [3], [7]-[9] and biased association [10]-[12]. The three metrics used for the selection of serving BS are (i) BS-mobile station (MS) distances, (ii) received SINR, and (iii) biasedSINR or biased-location. In these studies, the locations of BSs in each tier are modeled with a homogeneous Poisson point process (PPP). The PPP [14] has extensively been used to model distributions of BSs and MSs [3]-[12], [15], [16].

\section{A. Prior related research}

In the closest-BS policy, the serving BS is the closest one. Essentially, this policy ensures the highest areaaveraged signal strength with the variation of the signal strength due to small- and large-scale fading averaged out. Reference [6] investigates the coverage probability, normalized per user mean bit rate and coverage gain (and mean rate loss) from static frequency re-use on the downlink of a single-tier network assuming spatial distribution of BSs follows a homogeneous PPP. The uplink

${ }^{1}$ node $=$ BS or access point, user $=$ mobile station $(\mathrm{MS})$ 
performance with per-mobile power control is investigated in [4]. The downlink performance of multi-tier networks is investigated in [5]. Multiple tier BSs have different transmitted powers, supported data rates, and deployment densities. The spatial distribution of BSs in each tier is modeled using an independent homogeneous PPP.

In highest-SINR policy, the serving $\mathrm{BS}$ is the one offering the highest SINR. When BSs are fully loaded, transmitting and receiving packets on all their radio resource (time-frequency) blocks at all times, this strategy maximizes the sum throughput [2]. Performance of multitier networks under this policy is investigated in [3] and [8] assuming a system model similar to that of [5]. In [3], Dhillon et al. show that under this policy the coverage probability of an open access interference-limited network does not depend upon the number of tiers or the density of BSs when target threshold SIRs are the same for all the tiers. Reference [7] investigates the downlink coverage probability of a single-tier network under highest-SINR policy by characterizing the distribution of BSs with a non-homogeneous PPP.

In multi-tier networks, the area covered by each type of BS varies significantly. For example, a macro-BS will provide umbrella coverage for an area covered by many pico-BSs and femto access points (femto-APs). Due to this reason, BSs with smaller coverage areas, such as pico-BSs or femto-APs, will have fewer active users and hence will be lightly loaded compared to macro-BSs [2]. Therefore, smart cell association policies should be capable of offloading users from highly loaded BSs to lightly loaded ones so that BSs offer them the best user-perceived rate [2]. This goal is achieved by introducing a bias into the association policy to account for the load of each type of BS. Performance of SINR-based biased association policies is investigated in [10], [11]. In [12], Mukherjee investigates the downlink performance of both SINR- and locationbased biased association policies.

In [17], [18] Wang and Reed propose and investigate the performance of equivalent received power connectivity (ERPC) policy for multi-tier networks. In this policy a mobile user connects to the BS, from which it receives maximum equivalent power (received power in a given tier averaged over small- and large-scale fading, and divided by target SINR for that tier). When target SINRs are the same across multiple tiers, this policy is equivalent to the closest-BS association.

\section{B. Motivation and our contribution}

Our contribution is motivated by the following two key factors.

1) In large cellular networks channel state information available to an MS usually limited to only a few nodes [16]. Thus, the association policy must rely only on this limited information.

2) Due to the power-law path loss, the area-averaged received signal strength decays quickly with the distance between transmitter and receiver. In future generations of networks, this path loss may even increase with lower BS antennas and the use of higher frequencies. Therefore, higher the area-averaged received power from a BS to a user, higher the probability that it will provide the highest instantaneous SINR for the user.

These factors suggest that search for the best serving BS should be limited to a set of BSs providing the areaaveraged received power (from here onward referred to as average received power) above a certain value. Motivated by these factors, we propose that a given user is served by the highest instantaneous SIR (from here onward referred to as SIR) BS from among BSs providing average received power above a predetermined value $P_{t h}$. Thus, this policy requires only the instantaneous SIR and the average received power of a limited number of BSs, but not of the full network. The conventional highest-SIR association [3] can be considered as a special case of the new policy, i.e., when $P_{t h} \rightarrow 0$. We consider two methods to determine $P_{t h}$ : (i) selecting a fixed value and (ii) iterative selection of $P_{t h}$. We show that iterative selection of $P_{t h}$ considerably reduces the number of BSs to be tracked and hence the complexity of the cell association process.

This policy is also extended to two-tier macro-pico deployments. These extensions assume different conditions for the availability of SIR and average received power of the pico-BSs: (i) both SIR and average received power are available, (ii) only the average received power is available. In multi-tier networks, BSs with smaller coverage areas, such as pico-BSs or femto-APs, will necessarily serve fewer users and hence will be lightly loaded compared to macroBSs [2]. Therefore, a smart cell association policy should be capable of off-loading user traffic from highly loaded BSs to lightly loaded ones. We show that the proposed policy enables user off-loading in two-tier networks.

Our earlier work [19] differs from this study in three ways. First, [19] investigates only the coverage probability of the proposed association policy, while the achievable rate analysis is not included. Secondly, the case when only the average received power is available in two-tier networks is not considered in [19]. Thirdly, only in this work we compare the coverage probability of the proposed cell association policy in two-tier networks with that of the maximum biased instantaneous received power association [10].

Notation: $\|x\|$ is the Euclidean distance to point $x$ from the origin. The probability of event $A$ is $\operatorname{Pr}(A) . \mathbb{E}(\cdot)$ is the expectation operation. The Laplace transform of random variable $X$ is $\mathcal{L}_{X}(s)=\mathbb{E}_{X}\left[e^{-s X}\right] . \Gamma_{i n}(n, a)=$ $\int_{a}^{\infty} t^{n-1} e^{-t} d t$ is the incomplete Gamma function and its inverse is $\Gamma_{\text {in }}^{-1}(\cdot, \cdot) \cdot{ }_{2} F_{1}[a, b ; c ; z]=\frac{1}{B(b, c-b)} \int_{0}^{1} t^{b-1}(1-$ $t)^{c-b-1}(1-t z)^{-a} d t$, Re $c>\operatorname{Re} b>0$ [20, eq. 9.111] is the Gauss's hypergeometric function, where $B(\beta, \gamma)=$ $\int_{0}^{1} t^{\beta-1}(1-t)^{\gamma-1} d t[20$, eq. 8.380.1] is the beta function.

\section{System Model: Single Tier Networks}

Consider downlink of a network with BSs distributed in $\mathbb{R}^{d}, d \in\{2,3\}$ according to a homogeneous $\operatorname{PPP} \Phi$ with 


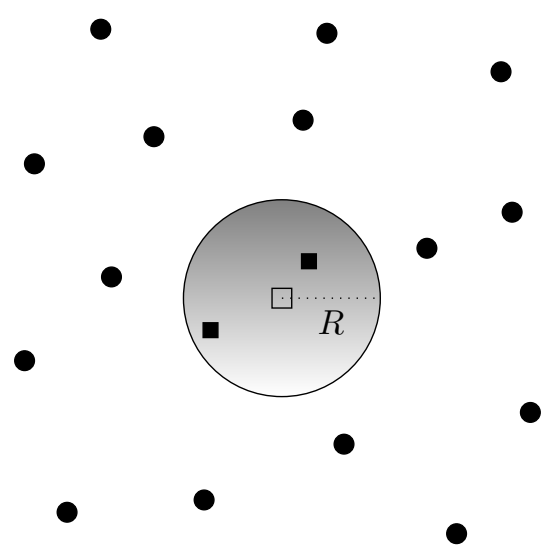

Fig. 1. Randomly distributed BSs in $\mathbb{R}^{2}$ with MS at the center. Black squares - accessible BSs, black circles - inaccessible BSs.

intensity $\lambda$ [14], [15]. Multi-user downlink transmission is assumed. However, transmission to each user within a given cell occurs on a different time-frequency resource block; the resource blocks within each cell are orthogonal. Universal time-frequency channel reuse and a fully loaded network are also assumed. Hence, the worst intercell interference case is considered. Every BS transmits with power $P_{t}$. Rayleigh fading (with envelope power normalized to one) is assumed along with path loss. We will use the simplified power-law path loss model, where the received power $P_{r}$ at a distance of $r$ from the transmitter is given as $P_{r}=P_{t} r^{-\alpha} . \alpha>d$ is the path loss exponent. The condition $\alpha>d$ is necessary to maintain a finite received power at each MS. Background thermal noise is ignored due to its negligible impact in interference-limited networks. In the proposed cell association policy serving BS is the one, which provides the highest SIR among all BSs meeting average received power requirement (from here onward referred to as candidate BSs). Since simplified path loss model is assumed, $P_{t h}$ defines a maximum distance $R=\left(\frac{P_{t}}{P_{t h}}\right)^{\frac{1}{\alpha}}$ a candidate BSs can be located from an MS (see Fig. 1). The highest-SIR association [3] is a special case of the new policy when $P_{t h} \rightarrow 0$ or equivalently $R \rightarrow \infty$.

In practice, MS-BS association is based on received signal strength indicator (RSSI) measurements from all available BSs (BSs that provide a meaningful RSSI). The association scheme proposed in this paper can be implemented by imposing a cut-off threshold value for the area-averaged RSSI to select a subset of BSs out of all the available BSs, and then choosing the BS with the highest instantaneous RSSI to serve a given MS. With that only the instantaneous RSSI of the selected subset of BSs and the average RSSI of available BSs need to be tracked. The selected subset of BSs should be updated periodically to account for the mobility of the MS and for changes in the radio wave propagation environment with time.

\section{Coverage Probability Analysis: Single-Tier NETWORK}

In this section first the coverage probability of a singletier network is derived. Subsequently two methods for the selection of $P_{t h}$ for cell association are presented.

\section{A. Coverage Probability Analysis}

Without loss of generality, we consider an MS located at the center of $\mathbb{R}^{d}$. As described in Section II, the minimum average received power requirement for candidate BSs divides the original space $\mathbb{R}^{d}$, over which the BSs are distributed, into two disjoint sub-spaces: $S_{1}=\left\{x \in \mathbb{R}^{d}\right.$ : $\|x\| \leq R\}$ and $S_{2}=\left\{x \in \mathbb{R}^{d}:\|x\|>R\right\}$, where $R=\left(\frac{P_{t}}{P_{t h}}\right)^{\frac{1}{\alpha}}$. Candidate BSs are distributed in the space $S_{1}$. According to the restriction theorem [21], BSs residing in $S_{1}$ and $S_{2}$ form two independent PPPs: $\Phi_{1}$ and $\Phi_{2}$, respectively. Assuming the MS is connected to a BS at point $y \in \Phi_{1}^{2}$, SIR of the MS can be written as

$$
\operatorname{SIR}(y)=\frac{\|y\|^{-\alpha} h_{y}}{\sum_{w \in \Phi_{1 \backslash y}}\|w\|^{-\alpha} h_{w}+\sum_{z \in \Phi_{2}}\|z\|^{-\alpha} h_{z}},
$$

where $h_{y}, h_{w}$, and $h_{z}$ represent the power gains due to multipath fading (fading coefficients). $\Phi_{1 \backslash y}$ denotes $\Phi_{1}$ excluding BS $y$. The resulting SIR at the MS is given by $S I R=\max _{y \in \Phi_{1}}\{S I R(y)\}$. An MS is considered to be in coverage, when its SIR equals or exceeds a given threshold $T$. Using a similar approach to that in [3], the coverage probability $P_{c}(T)$ under the new cell association policy can be expressed as

$$
\begin{aligned}
P_{c}(T) & =\operatorname{Pr}\left(\bigcup_{y \in \Phi_{1}} \operatorname{SIR}(y) \geq T\right) \\
& \leq \mathbb{E}\left[\sum_{y \in \Phi_{1}} \mathbf{1}(\operatorname{SIR}(y) \geq T)\right],
\end{aligned}
$$

where the indicator function $\mathbf{1}(x)=1$, if $x$ holds, else 0 . The bound is the union bound on $P_{c}(T)$. Using Lemma 1 of [3], it can be easily shown that the union bound is exact for threshold SIRs $T>0 \mathrm{~dB}$ and is an upper bound for $T<0$ dB. Now $(2)$ can be written as [19]

$$
\begin{aligned}
P_{c}(T) & \stackrel{a}{\leq} \lambda \int_{S_{1}} \operatorname{Pr}\left(h_{y} \geq \frac{T\left(I_{1 \backslash y}+I_{2}\right)}{\|y\|^{-\alpha}}\right) d y \\
& \leq \lambda \int_{S_{1}} \mathbb{E}_{I_{1 \backslash y}+I_{2}}\left[\exp \left(-\frac{T\left(I_{1 \backslash y}+I_{2}\right)}{\|y\|^{-\alpha}}\right)\right] d y \\
& \leq \lambda \int_{S_{1}} \mathcal{L}_{I_{1 \backslash y}+I_{2}}\left(\frac{T}{\|y\|^{-\alpha}}\right) d y,
\end{aligned}
$$

where (a) follows from the Campbell-Mecke theorem [14] and (b) follows from $h_{x} \sim \exp (1)$. (c) follows form the definition of the Laplace transform. $I_{1 \backslash y}=$

\footnotetext{
${ }^{2}$ With slight abuse of notation we will use $y$ to denote both the location of MS and MS itself. Similarly for $w$ and $z$.
} 
$\sum_{w \in \Phi_{1 \backslash y}}\|w\|^{-\alpha} h_{w}$ is the total interference power from all the BSs in $\Phi_{1}$, when MS is connected to BS at the point $y$ and all the BSs transmit with unity power. $I_{2}=\sum_{z \in \Phi_{2}}\|z\|^{-\alpha} h_{z}$ is the total interference power from $\Phi_{2}$ when all BSs transmit with unity power. $\mathcal{L}_{I_{1 \backslash y}+I_{2}}(s)$ can be derived as shown in the following [19].

Since the fading coefficients are independent and two PPPs $\Phi_{1}$ and $\Phi_{2}$ are independent of each other and independent of the fading processes, $I_{1 \backslash y}$ and $I_{2}$ are statistically independent. Therefore,

$$
\begin{aligned}
\mathcal{L}_{I_{1 \backslash y}+I_{2}}(s)= & \mathbb{E}_{\Phi_{1}, h_{w}}\left[\exp \left(-s \sum_{w \in \Phi_{1} \backslash y}\|w\|^{-\alpha} h_{w}\right)\right] \\
& \times \mathbb{E}_{\Phi_{2}, h_{z}}\left[\exp \left(-s \sum_{z \in \Phi_{2}}\|z\|^{-\alpha} h_{z}\right)\right] .
\end{aligned}
$$

Since Rayleigh fading is assumed $h_{w} \sim \exp (1)$ and $h_{z} \sim$ $\exp (1)$. Therefore,

$$
\begin{aligned}
& \mathcal{L}_{I_{1 \backslash y}+I_{2}}(s)=\mathbb{E}_{\Phi_{1}}\left[\prod_{w \in \Phi_{1} \backslash y} \frac{1}{1+s\|w\|^{-\alpha}}\right] \\
& \times \mathbb{E}_{\Phi_{2}}\left[\prod_{z \in \Phi_{2}} \frac{1}{1+s\|z\|-\alpha}\right] .
\end{aligned}
$$

Using the Slivnyak-Mecke theorem [14] along with the definition of probability generating functional of PPP [14] we get:

$$
\begin{aligned}
\mathcal{L}_{I_{1 \backslash y}+I_{2}}(s) & =\exp \left[-\lambda \int_{S_{1}}\left(1-\frac{1}{1+s\|w\|^{-\alpha}}\right) d w\right] \\
& \times \exp \left[-\lambda \int_{S_{2}}\left(1-\frac{1}{1+s\|z\|^{-\alpha}}\right) d z\right] \\
& =\exp \left[-\lambda \int_{\mathbb{R}^{d}}\left(1-\frac{1}{1+s\|u\|^{-\alpha}}\right) d u\right] .
\end{aligned}
$$

Converting from Cartesian to polar/spherical coordinates, for $d$-dimensional networks (6) can be written as

$$
\begin{aligned}
\mathcal{L}_{I_{1 \backslash y}+I_{2}}(s) & =\exp \left[-\kappa_{d} \pi \lambda \int_{0}^{\infty} r^{d-1}\left(1-\frac{1}{1+s r^{-\alpha}}\right) d r\right] \\
& =\exp \left[\frac{-\kappa_{d} \pi^{2} \lambda s^{\frac{d}{\alpha}}}{\alpha \sin \left(\frac{d \pi}{\alpha}\right)}\right],
\end{aligned}
$$

where $\kappa_{2}=2$, and $\kappa_{3}=4$. Converting (3) from Cartesian to polar/spherical coordinates and substituting (7) in this new expression, $P_{c}(T)$ for $d$-dimensional networks can be expressed as

$$
P_{c}(T) \leq\left[1-\exp \left(\frac{-\kappa_{d} \pi^{2}\left(\frac{P_{t} T}{P_{t h}}\right)^{\frac{d}{\alpha}} \lambda}{\alpha \sin \left(\frac{d \pi}{\alpha}\right)}\right)\right] \frac{\alpha \sin \left(\frac{d \pi}{\alpha}\right)}{d \pi T^{\frac{d}{\alpha}}} .
$$

According to (8), the impact of $\lambda$ on $P_{c}(T)$ diminishes as $P_{t h} \rightarrow 0$. A similar observation is made for 2 -D networks in Corollary 1 of [3].

\section{B. Selection of Minimum Average Received Power $P_{t h}$}

We know that if $P_{t h}$ is too large, no BS is likely to be selected as a candidate BS, which will put the MS into outage. Conversely, if $P_{t h}$ is too small, too many BSs will be selected as candidate BSs, increasing the complexity of the association. Thus, $P_{t h}$ should be chosen such that more than $n(\geq 1)$ BSs provide average received power above $P_{t h}$ with a reasonably high probability $q$. For example, $n=2$ and $q=0.95$ will guarantee that two candidate BSs are available with a probability of 0.95 . For a network with a homogeneous PPP distribution of BSs, $P_{t h}$ for selected set of values for $n$ and $q$ can be computed as follows.

Let $P_{n}$ be the $n$th highest average received power from BSs. Since the average received power only depends on the path loss and BS transmitted power, which is assumed to be constant, $P_{n}$ is the average received power from $n$th closest BS to the MS. The cumulative distribution function (CDF) of the random variable $P_{n}$ is given by

$$
F_{P_{n}}(z)=\operatorname{Pr}\left[P_{t} r_{n}^{-\alpha} \leq z\right]=1-\operatorname{Pr}\left[r_{n} \leq\left(\frac{P_{t}}{z}\right)^{\frac{1}{\alpha}}\right]
$$

where $r_{n}$ is the Euclidean distance between an MS and its $n$th closest BS in $\mathbb{R}^{d}$. The CDF of $r_{n}$ is given by [22]

$$
F\left(r_{n}\right)=1-\frac{\Gamma_{i n}\left(n, \lambda c_{d} r_{n}^{d}\right)}{(n-1) !}, 0<r_{n}<\infty
$$

where $c_{2}=\pi, c_{3}=4 \pi / 3$. Substituting (10) in (9),

$$
F_{P_{n}}(z)=\frac{\Gamma_{i n}\left(n, \lambda c_{d}\left(\frac{P_{t}}{z}\right)^{\frac{d}{\alpha}}\right)}{(n-1) !}, 0<z<\infty
$$

Therefore, in $\mathbb{R}^{d}$, for a given number of BSs $n$ and probability $q=1-F_{P_{n}}\left(P_{t h}\right), P_{t h}$ can be computed as

$$
P_{t h}=P_{t}\left[\frac{\lambda c_{d}}{\Gamma_{i n}^{-1}(n,(n-1) !(1-q))}\right]^{\frac{\alpha}{d}}
$$

Improving $P_{c}$ by iterative decrease of $P_{t h}$

The second way to select $P_{t h}$ is to use an adaptive update. The problem of selecting too many BSs as candidate BSs can be alleviated by initializing the association with a large value for $P_{t h}$ (equivalently, small $n$ and $q$ ), and decreasing it iteratively. For example, a system may select the initial minimum association received power $P_{t h}^{(0)}$ by selecting lower values for $n$ and $q, n^{(0)}$ and $q^{(0)}$ (e.g., $n^{(0)}=1$ and $q^{(0)}=0.9$, which guarantees at least one BS given $P_{t h}^{(0)}, 90 \%$ of the time). If no BS to associate with is found, $P_{t h}$ can be decreased iteratively $N$ times (e.g., with $N=2$, new minimum association received power after 1st iteration $P_{t h}^{(1)}$ is selected with $n^{(1)}=1$ and $q^{(1)}=0.99$, which guarantees one BS $99 \%$ of the time; in the second iteration, $P_{t h}^{(2)}$ with $n^{(2)}=1$ and $\left.q^{(2)}=0.999\right)$. This process is implemented by Algorithm 1 listed below. In Section VII it is shown that the iterative selection of $P_{t h}$ considerably reduces the number of BSs to be scanned, therefore it lowers the complexity of the association process. 


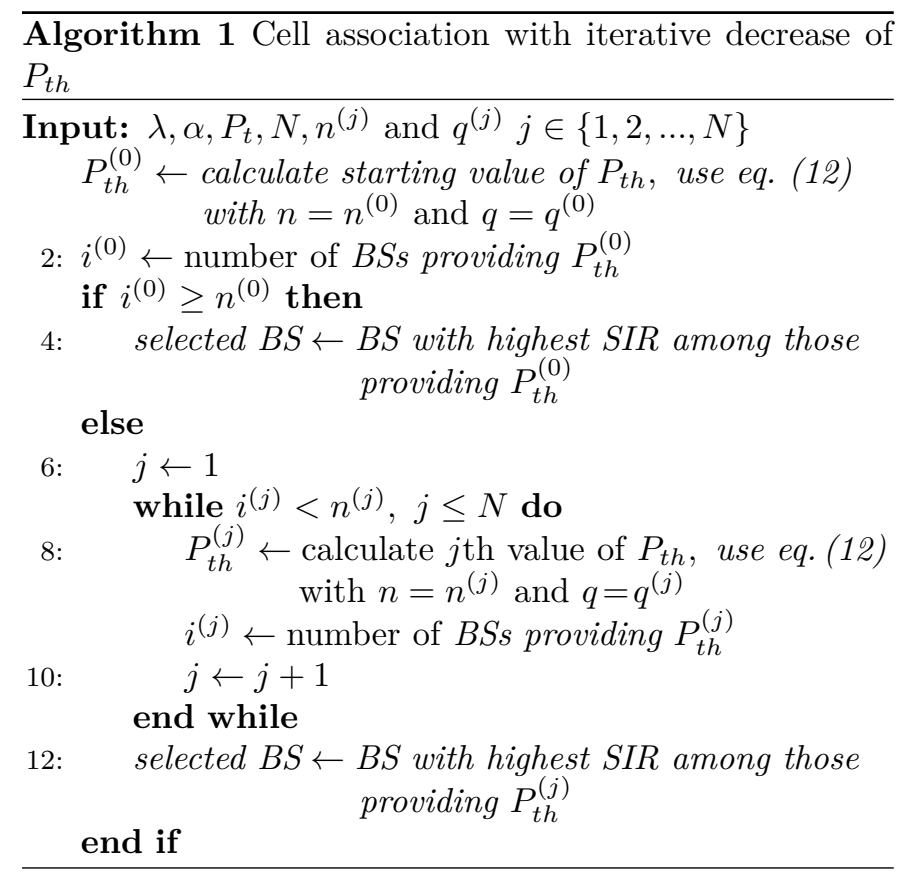

\section{Extensions for Two-Tier Heterogeneous NETWORKS}

Consider a two-tier network consisting of macro- and pico-BSs. Two cases are considered for the availability of average received power and SIR information of pico-BSs: (i) both SIR information and average received power are available, (ii) only the average received power is available.

For case (i), association policy attempts to select a picoBS providing average received power exceeding $P_{t h}$. If only one pico-BS is available, the MS associates with it. When more than one pico-BSs are available, one providing the highest instantaneous SIR is selected. If the average received powers from all the pico-BSs are lower than $P_{t h}$, the macro-BS providing the highest average SIR is selected as the serving BS. This is equivalent to associating with the closest macro-BS. The ability to manage user offloading to small cells by $P_{t h}$ is among the advantages of this association policy. For example, $70 \%$ of users will be served by pico-BSs, when $P_{t h}$ is selected with $q=0.7$ and $n=1$ ( $70 \%$ of the time there is at least one pico-BS providing average received power exceeding $\left.P_{t h}\right)$. For case (ii), each MS connects to the pico-BS providing maximum average received power (equivalently the BS providing maximum average SIR), if its received power exceeds $P_{t h}$. If this fails, it is connected to the macro-BS providing the highest average SIR.

\section{Two-Tier Network: Network Parameters}

We characterize the spatial distribution of macro- and pico-BSs by two independent homogeneous PPPs $\Phi_{m}$ and $\Phi_{p}$ with intensities $\lambda_{m}$ and $\lambda_{p}$, respectively. Macro-BSs transmit with power $P_{m}$, while pico-BSs transmit with power $P_{p}$. All the links are assumed to be subject to path loss (as in Section II) and multipath fading. Path loss exponents of macro-BSs and pico-BSs are given by $\alpha_{m}$ and $\alpha_{p}\left(\alpha_{m}, \alpha_{p}>2\right)$. Target SIR thresholds of macroBSs and pico-BSs are $T_{m}$ and $T_{p}$, respectively. Multi-user downlink transmission is assumed, as described in detail in the first paragraph of Section II.

\section{Coverage Probability Analysis: Two-Tier NETWORKS}

This section presents the coverage probability analysis for two cases, namely (i) both instantaneous SIR and average received power of pico-BSs are available (ii) only average received power is available. Without loss of generality the coverage probability of an MS located at the center of the network is considered.

\section{A. Both Instantaneous SIR and Average Received Power Available}

When both SIR and average received power of pico-BSs are available, the proposed association policy selects the pico-BS providing the maximum instantaneous SIR from those providing average received power exceeding $P_{t h}$. If no pico-BS is found to provide such average power, the macro-BS with the highest average SIR is selected as the serving BS. Using the theorem on total probability the coverage probability can be expressed as

$$
P_{c}\left(T_{m}, T_{p}\right)=P_{v} P_{c}^{\text {macro }}\left(T_{m}\right)+\left(1-P_{v}\right) P_{c \mid r_{u}<R_{p}}^{\text {pico }}\left(T_{p}\right),
$$

where $r_{u}=\|u\|$ is the distance between the closest picoBS $u \in \Phi_{p}$ and the MS. $R_{p}=\left(\frac{P_{p}}{P_{t h}}\right)^{\frac{1}{\alpha_{p}}} . P_{c}^{\text {macro }}\left(T_{m}\right)$ is the coverage probability when the MS is served by the macro-BS providing the highest average SIR given that there is no pico-BS providing $P_{t h} . P_{c \mid r_{u}<R_{p}}^{\text {pico }}\left(T_{p}\right)$ gives the coverage probability, when the MS is served by the highest SIR pico-BS out of those providing $P_{t h}$. $P_{v}$ represents the probability that no pico-BS is found to provide $P_{t h}$ at the MS. $P_{v}$ can be derived as

$$
P_{v}=\operatorname{Pr}\left(P_{p} r_{u}^{-\alpha_{p}}<P_{t h}\right)=\operatorname{Pr}\left(r_{u}>\left(\frac{P_{p}}{P_{t h}}\right)^{\frac{1}{\alpha_{p}}}\right),
$$

$\operatorname{Pr}\left(r_{u}>\left(\frac{P_{p}}{P_{t h}}\right)^{\frac{1}{\alpha_{p}}}\right)$ is the probability that no pico-BS is found within distance $\left(\frac{P_{p}}{P_{t h}}\right)^{\frac{1}{\alpha_{p}}}$ from the MS. Since picoBSs are distributed according to a PPP with intensity $\lambda_{p}$,

$$
P_{v}=\exp \left(-\lambda_{p} \pi\left(\frac{P_{p}}{P_{t h}}\right)^{\frac{2}{\alpha_{p}}}\right)
$$

Using a similar approach to the derivation of (2) and (3), $\hat{P}_{c \mid r_{u}<R_{p}}^{\text {pico }}\left(T_{p}\right)=\left(1-P_{v}\right) P_{c \mid r_{u}<R_{p}}^{\text {pico }}\left(T_{p}\right)$ can be derived as follows [19]:

$$
\begin{gathered}
\hat{P}_{c \mid r_{u}<R_{p}}^{\text {pico }}\left(T_{p}\right) \leq \lambda_{p} \int_{\mathcal{A}_{\mathcal{R}}} \operatorname{Pr}\left(h_{y} \geq \frac{T_{p}\left(I_{p \backslash y}+I_{m}\right)}{P_{p}\|y\|^{-\alpha_{p}}}\right) d y \\
\quad \stackrel{a}{\leq} \lambda_{p} \int_{\mathcal{A}_{\mathcal{R}}} \mathcal{L}_{I_{p \backslash y}+I_{m}}\left(\frac{T_{p}}{P_{p}\|y\|^{-\alpha_{p}}}\right) d y
\end{gathered}
$$




$$
\leq 2 \pi \lambda_{p} \int_{0}^{R_{p}} r \mathcal{L}_{I_{p \backslash y}+I_{m}}\left(\frac{T_{p}}{P_{p} r^{-\alpha_{p}}}\right) d r
$$

where $\mathcal{A}_{R}$ represents the circular region around the MS with radius $R_{p}$. Step (a) follows from the Rayleigh fading assumption. $I_{p \backslash y}$ represents the aggregate interference from pico-BSs when MS is served by pico-BS located at $y \in \Phi_{p} . I_{m}$ represents the aggregate interference from macro-BSs. Similarly to (3), the expression (16) is exact for threshold SIRs $T_{p}>0 \mathrm{~dB}$ and is an upper bound for $T_{p}<0 \mathrm{~dB}$. Since fading coefficients are independent and identically distributed (i.i.d.) and the fading processes are independent from PPPs $\mathcal{L}_{I_{p \backslash y}+I_{m}}(s)=\mathcal{L}_{I_{p \backslash y}}(s) \times \mathcal{L}_{I_{m}}(s)$. $\mathcal{L}_{I_{p \backslash y}}(s)$ can be obtained by replacing $s, \lambda$, and $\alpha$ in (7) with $s P_{p}, \lambda_{p}$, and $\alpha_{p}$, respectively.

$$
\mathcal{L}_{I_{p \backslash y}}(s)=\exp \left[\frac{-2 \pi^{2} \lambda_{p}\left(s P_{p}\right)^{\frac{2}{\alpha_{p}}}}{\alpha_{p} \sin \left(\frac{2 \pi}{\alpha_{p}}\right)}\right] .
$$

Following similar steps to those in the derivation of (7), $\mathcal{L}_{I_{m}}(s)$ can be expressed as

$$
\begin{aligned}
\mathcal{L}_{I_{m}}(s) & =\mathbb{E}_{\Phi_{m}}\left[\prod_{x \in \Phi_{m}} \mathbb{E}_{h_{x}}\left[\exp \left(-s P_{m}\|x\|^{-\alpha_{m}} h_{x}\right)\right]\right] \\
& =\exp \left[\frac{-2 \pi^{2} \lambda_{m}\left(s P_{m}\right)^{\frac{2}{\alpha_{m}}}}{\alpha_{m} \sin \left(\frac{2 \pi}{\alpha_{m}}\right)}\right] .
\end{aligned}
$$

$P_{c}^{\text {macro }}\left(T_{m}\right)$ can be derived as shown in the following. When MS is served by the highest average SIR macro-BS $z \in \Phi_{m}, \mathrm{SIR}$ is given by

$$
\operatorname{SIR}(z)=\frac{P_{m} r_{z}^{-\alpha_{m}} h_{z}}{\sum_{x \in \Phi_{m} \backslash z} P_{m}\|x\|^{-\alpha_{m}} h_{x}+\sum_{y \in \Phi_{p},\|y\|>R_{p}} P_{p}\|y\|^{-\alpha_{p}} h_{y}},
$$

where distance between MS and the highest average SIR macro-BS (equivalently, the closest macro-BS) is denoted by $r_{z}=\|z\|$. Therefore, $P_{c}^{\text {macro }}\left(T_{m}\right)$ is given by [19]:

$$
\begin{aligned}
P_{c}^{\text {macro }} & \left(T_{m}\right)=\operatorname{Pr}\left[\frac{P_{m} r_{z}^{-\alpha_{m}} h_{z}}{I_{m \backslash z}+I_{p \backslash \mathcal{A}_{\mathcal{R}}}} \geq T_{m}\right] \\
& =\int_{0}^{\infty} \operatorname{Pr}\left[\frac{P_{m} r_{z}^{-\alpha_{m}} h_{z}}{I_{m \backslash z}+I_{p \backslash \mathcal{A}_{\mathcal{R}}}} \geq T_{m} \mid r_{z}=t\right] f_{r_{z}}(t) d t \\
& =\int_{0}^{\infty} \operatorname{Pr}\left[h_{z} \geq \frac{T_{m}\left(I_{m \backslash z}+I_{p \backslash \mathcal{A}_{\mathcal{R}}}\right)}{P_{m} r_{z}^{-\alpha_{m}}} \mid r_{z}=t\right] f_{r_{z}}(t) d t \\
& \stackrel{a}{=} \int_{0}^{\infty} \mathcal{L}_{I_{m \backslash z}+I_{p \backslash \mathcal{A}_{\mathcal{R}}} \mid r_{z}=t}\left(\frac{T_{m}}{P_{m} t^{-\alpha_{m}}}\right) f_{r_{z}}(t) d t .
\end{aligned}
$$

Here, $\quad I_{m \backslash z}=\sum_{x \in \Phi_{m \backslash z}} P_{m}\|x\|^{-\alpha_{m}} h_{x} \quad$ and $I_{p \backslash \mathcal{A}_{\mathcal{R}}}=\sum_{y \in \Phi_{p},\|y\|>R_{p}} P_{p}\|y\|^{-\alpha_{p}} h_{y}$. Step (a) follows from the Rayleigh fading assumption. The probability density function of $r_{z}$ is given by [22]: $f_{r_{z}}(t)=2 \pi \lambda_{m} t \exp \left(-\lambda_{m} \pi t^{2}\right), t>0 . \mathcal{L}_{I_{m \backslash z}+I_{p} \backslash \mathcal{A}_{\mathcal{R}}}(s)$ can be derived as shown in the following [19].

$$
\begin{aligned}
& \mathcal{L}_{I_{m \backslash z}+I_{p \backslash \mathcal{A}_{\mathcal{R}}} \mid r_{z}=t}(s) \stackrel{a}{=} \mathcal{L}_{I_{m \backslash z} \mid r_{z}=t}(s) \times \mathcal{L}_{I_{p \backslash \mathcal{A}_{\mathcal{R}}}}(s) \\
& \stackrel{b}{=} \mathbb{E}_{\Phi_{m}, h_{x}}\left[\exp \left(-s \sum_{x \in \Phi_{m \backslash z}} P_{m}\|x\|^{-\alpha_{m}} h_{x}\right)\right]
\end{aligned}
$$

$$
\begin{aligned}
& \times \mathbb{E}_{\Phi_{p}, h_{y}}\left[\exp \left(-s \sum_{y \in \Phi_{p},\|y\|>R_{p}} P_{p}\|y\|^{-\alpha_{p}} h_{y}\right)\right] \\
& \stackrel{c}{=} \mathbb{E}_{\Phi_{m}}\left[\prod_{x \in \Phi_{m \backslash z}} \mathbb{E}_{h_{x}}\left[\exp \left(-s P_{m}\|x\|^{-\alpha_{m}} h_{x}\right)\right]\right] \\
& \times \mathbb{E}_{\Phi_{p}}\left[\prod_{y \in \Phi_{p},\|y\|>R_{p}} \mathbb{E}_{h_{y}}\left[\exp \left(-s P_{p}\|y\|^{-\alpha_{p}} h_{y}\right)\right]\right] \\
& \stackrel{d}{=} \mathbb{E}_{\Phi_{m}}\left[\prod_{x \in \Phi_{m \backslash z}} \frac{1}{1+\left.s P_{m}\|x\|\right|^{-\alpha_{m}}}\right] \\
& \times \mathbb{E}_{\Phi_{p}}\left[\prod_{y \in \Phi_{p},\|y\|>R_{p}} \frac{1}{1+\left.s P_{p}\|y\|\right|^{-\alpha_{p}}}\right] \\
& \stackrel{e}{=} \exp \left[-2 \pi \lambda_{m} \int_{t}^{\infty} u\left(1-\frac{1}{1+s P_{m} u^{-\alpha_{m}}}\right) d u\right] \\
& \times \exp \left[-2 \pi \lambda_{p} \int_{R_{p}}^{\infty} v\left(1-\frac{1}{1+s P_{p} v^{-\alpha_{p}}}\right) d v\right] \\
& =\exp \left[\frac{-2 \pi \lambda_{m} P_{m} t^{2-\alpha_{m}} s{ }_{2} F_{1}\left[1, \frac{\alpha_{m}-2}{\alpha_{m}} ; 2-\frac{2}{\alpha_{m}}, \frac{-P_{m} s}{t^{\alpha_{m}}}\right]}{\alpha_{m}-2}\right] \\
& \times \exp \left[\frac{-2 \pi \lambda_{p} P_{p}^{\frac{2}{\alpha_{p}}} P_{t h}^{1-\frac{2}{\alpha_{p}}} s{ }_{2} F_{1}\left[1, \frac{\alpha_{p}-2}{\alpha_{p}} ; 2-\frac{2}{\alpha_{p}},-s P_{t h}\right]}{\alpha_{p}-2}\right] .
\end{aligned}
$$

Steps (a) and (c) follow from the fact that fading coefficients are i.i.d. and two PPPs $\Phi_{m}$ and $\Phi_{p}$ are independent of each other and independent of the respective fading processes. (b) follows from the definition of the Laplace transform. (d) is due to the Rayleigh fading assumption. (e) follows from the definition of probability generating functional of PPP [14] and by converting from Cartesian to polar coordinates.

\section{B. Only Average Received Power Available}

When only average received powers of pico-BSs are available, each MS attempts to connect to the pico-BS with the highest average received power, if the average received power from it exceeds $P_{t h}$. Essentially, this is connecting to the closest pico-BS if it meets the minimum average received power requirement. If no pico-BS is found providing $P_{t h}$, MS associates with the macro-BS providing the highest average SIR. The coverage probability of the network for this case can be derived as follows. From the theorem on total probability

$$
P_{c}\left(T_{m}, T_{p}\right)=\left(1-P_{v}\right) \tilde{P}_{c \mid r_{u}<R_{p}}^{\text {pico }}\left(T_{p}\right)+P_{v} P_{c}^{\text {macro }}\left(T_{m}\right),
$$

where $\tilde{P}_{c \mid r_{u}<R_{p}}^{\text {pico }}\left(T_{p}\right)$ is the coverage probability, when the user is served by the pico-BS with the highest average received power provided that the average received power exceeds $P_{t h} . P_{v}$ and $P_{c}^{\text {macro }}\left(T_{m}\right)$ are given by $(15)$ and (20), respectively. Using the definition of conditional probability $\left(1-P_{v}\right) \tilde{P}_{c \mid r_{z}<R_{p}}^{\text {pico }}\left(T_{p}\right)$ can be expressed as

$$
\left(1-P_{v}\right) \tilde{P}_{c \mid r_{u}<R_{p}}^{\text {pico }}\left(T_{p}\right)=\operatorname{Pr}\left[\frac{P_{p} r_{u}^{-\alpha_{p}} h_{u}}{I_{p \backslash u}+I_{m}} \geq T_{p}, r_{u}<R_{p}\right]
$$




$$
=\int_{0}^{R_{p}} \operatorname{Pr}\left[h_{u}>\frac{T_{p}\left(I_{p \backslash u}+I_{m}\right)}{P_{p} r_{u}^{-\alpha_{p}}} \mid r_{u}=t\right] f_{r_{u}}(t) d t,
$$

where $I_{p \backslash u}$ is the total interference from all other pico-BSs when MS is connected to the pico-BS with the highest average received power provided that the average received power exceeds $P_{t h} . h_{u}$ is the fading power gain. $f_{r_{u}}(t)$ is the PDF of $r_{u}$, distance from the MS to the pico-BS with the highest average received power (equivalently, the closest pico-BS) from the MS. Since $h_{u} \sim \exp (1),(23)$ can be written as

$$
\begin{aligned}
\left(1-P_{v}\right) \tilde{P}_{c \mid r_{u}<R_{p}}^{\text {pico }}\left(T_{p}\right)= & 2 \pi \lambda_{p} \int_{0}^{R_{p}} t \mathcal{L}_{I_{p \backslash u}+I_{m} \mid r_{u}=t}\left(\frac{T_{p} t^{\alpha_{p}}}{P_{p}}\right) \\
& \times \exp \left(-\lambda_{p} \pi t^{2}\right) d t .
\end{aligned}
$$

where $\mathcal{L}_{I_{p \backslash u}+I_{m} \mid r_{u}=t}(s)$ represents the Laplace transform of $I_{p \backslash u \mid r_{u}=t}+I_{m}(s)$ with respect to $s$. Since $I_{p \backslash u \mid r_{u}=t}$ and $I_{m}$ are statistically independent, $\mathcal{L}_{I_{p \backslash u}+I_{m} \mid r_{u}=t}(s)=$ $\mathcal{L}_{I_{p \backslash u} \mid r_{u}=t}(s) \times \mathcal{L}_{I_{m}}(s) \cdot \mathcal{L}_{I_{m}}(s)$ is given by (18). Following a similar approach to the derivation of $\mathcal{L}_{I_{m \backslash z} \mid r_{z}=t}(s)$ in $(21), \mathcal{L}_{I_{p \backslash u} \mid r_{u}=t}(s)$ can be obtained as

$$
\begin{array}{r}
\mathcal{L}_{I_{p \backslash u} \mid r_{u}=t}(s)=\exp \left[-2 \pi \lambda_{p} \int_{t}^{\infty} y\left(1-\frac{1}{1+s P_{p} y^{-\alpha_{p}}}\right) d y\right] \\
=\exp \left[\frac{-2 \pi \lambda_{p} P_{p} t^{2-\alpha_{p}} s_{2} F_{1}\left[1, \frac{\alpha_{p}-2}{\alpha_{p}} ; 2-\frac{2}{\alpha_{p}}, \frac{-P_{p} s}{t^{\alpha p}}\right]}{\alpha_{p}-2}\right] .
\end{array}
$$

\section{Achievable Rate Analysis}

In this section, the average rate achievable by a randomly chosen MS in coverage is investigated. First, average rate of an MS in a single-tier network is derived. Subsequently, the derived expression is extended to twotier networks.

\section{A. Single-Tier Networks}

The average rate achievable by a randomly chosen MS in coverage in a single-tier network can be expressed as:

$$
R^{1 \text {-tier }}=\mathbb{E}_{S I R \mid S I R} \geq T[\ln (1+S I R)] .
$$

Here $S I R=\max _{y \in \Phi_{1}}\{\operatorname{SIR}(y)\}$ where, $\operatorname{SIR}(y)$ is given by (1). Since the expectation of any positive random variable $\mathrm{X}$ is given by $\mathbb{E}[X]=\int_{0}^{\infty} \operatorname{Pr}(x>z) d z$,

$$
\begin{aligned}
R^{1-\text { tier }} & =\int_{0}^{\infty} \operatorname{Pr}[\ln (1+S I R)>z \mid S I R \geq T] d z \\
& =\int_{0}^{\infty} \operatorname{Pr}\left[S I R>e^{z}-1 \mid S I R \geq T\right] d z .
\end{aligned}
$$

Using the definition of conditional probability

$$
\begin{aligned}
R^{1 \text {-tier }} & =\int_{0}^{\infty} \frac{\operatorname{Pr}\left[S I R>e^{z}-1, S I R \geq T\right]}{\operatorname{Pr}[S I R \geq T]} d z \\
& =\ln (1+T)+\int_{\ln (1+T)}^{\infty} \frac{\operatorname{Pr}\left[S I R>e^{z}-1\right]}{\operatorname{Pr}[S I R>T]} d z \\
& =\ln (1+T)+\frac{1}{P_{c}(T)} \int_{\ln (1+T)}^{\infty} P_{c}\left(e^{z}-1\right) d z
\end{aligned}
$$

where, $P_{c}(x)$ is the coverage probability in a single-tier network when threshold SIR is $x$, which is given by (8). In Section III-A, it was shown that (8) is an exact expression for $T \geq 0 \mathrm{~dB}$ and an upper bound when $T<0 \mathrm{~dB}$. Consequently, (28) is an exact expression for $T \geq 0 \mathrm{~dB}$ and a lower bound when $T<0 \mathrm{~dB}$.

\section{B. Two-Tier Networks}

The achievable rate of an MS in coverage in a two-tier network $R^{2 \text {-tier }}$ is analytically intractable when the SIR thresholds of two types of BSs are different. Therefore, we derive $R^{2 \text {-tier }}$ assuming SIR thresholds to be the same, i.e., $T_{m}=T_{p}=T$. Following a similar approach to the derivation of (28), $R^{2 \text {-tier }}$ has been derived as

$$
R^{2 \text {-tier }}=\ln (1+T)+\frac{1}{P_{c}(T, T)} \int_{\ln (1+T)}^{\infty} P_{c}\left(e^{z}-1, e^{z}-1\right) d z
$$

where, $P_{c}(x, y)$ is given by (13) or (22) depending on the availability of SIR/average received power information. Similarly to (28), (29) is an exact expression for $T_{m}, T_{p} \geq 0 \mathrm{~dB}$ and a lower bound when $T_{m}, T_{p}<0$ $\mathrm{dB}$.

\section{Numerical Results}

This section first considers a single-tier network with the proposed cell association policy. Its coverage probability is compared with those of the closest-BS and the highestSIR association policies. Secondly, the coverage probability of a two-tier network, which employs extensions of the new association policy, is investigated. Performance of the new policy is compared with those of the closest-BS, the highest-SIR and the maximum biased instantaneous received power [10] association policies. Finally the average rates of MSs in coverage of both single-tier and two-tier networks are investigated.

Fig. 2 shows the variation of $P_{c}$ in a single-tier network with the new policy. The analytical and simulation results are very close when $T>-2 \mathrm{~dB}$. Also, the coverage probability of the new policy is compared with those of the closest-BS and the highest-SIR policies. Interestingly, when $P_{t h}$ is selected appropriately (equivalently, $n$ and $q$ are appropriately selected), this policy outperforms the closest-BS association and performs similarly to the highest-SIR policy. For example, the new policy with $n=1$, and $q=0.99$ achieves similar performance as the highest-SIR one. Due to path loss, to achieve a higher SIR an MS needs to associate with a much closer BS. Therefore, as can be seen, the performances of all the policies converge to that of the closest-BS policy when threshold SIR is higher $(T>6 \mathrm{~dB})$.

Coverage probability improvement that can be achieved by adaptive selection of $P_{t h}$ is shown in Fig. 3. In the network considered, $P_{t h}^{(j)}, j \in\{0,1,2\}$ is selected with $\forall j: n^{(j)}=1, q^{(0)}=0.9, q^{(1)}=0.99$, and $q^{(2)}=0.999$. Therefore, $P_{t h}^{(0)}=-40.74 \mathrm{dBm}, P_{t h}^{(1)}=-46.01 \mathrm{dBm}$, and $P_{t h}^{(2)}=-49.09 \mathrm{dBm}$. Results show that $P_{c}$ with 


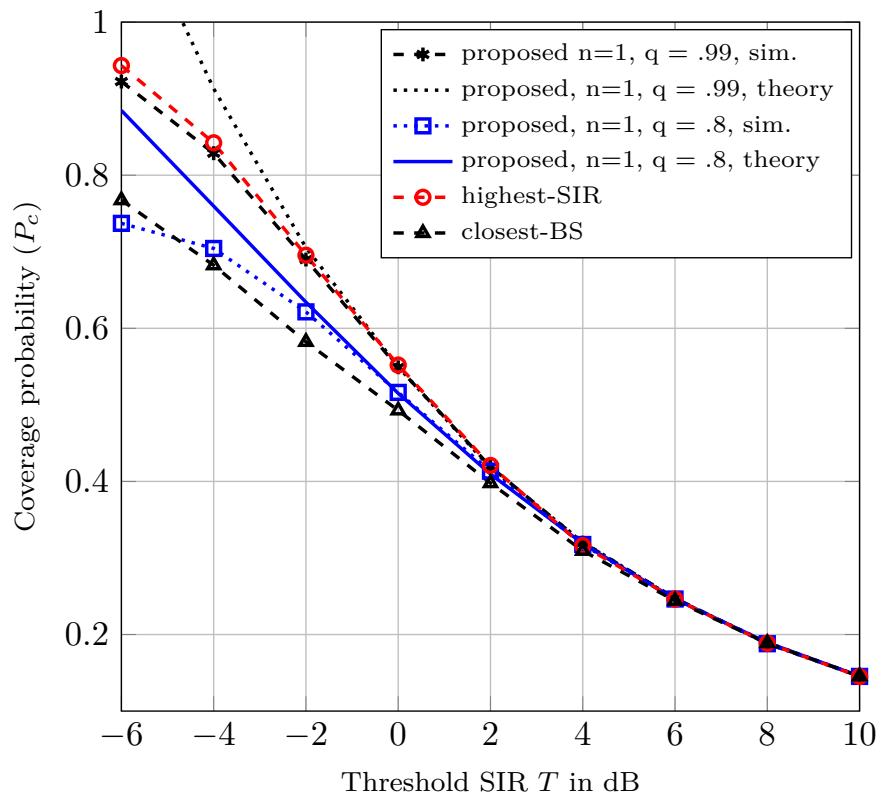

Fig. 2. Variation of $P_{c}$ in a single-tier network with T. $\alpha=3.5$, $\lambda=12 \times 10^{-6} \mathrm{~m}^{-2}, P_{t}=20 \mathrm{~W}$.

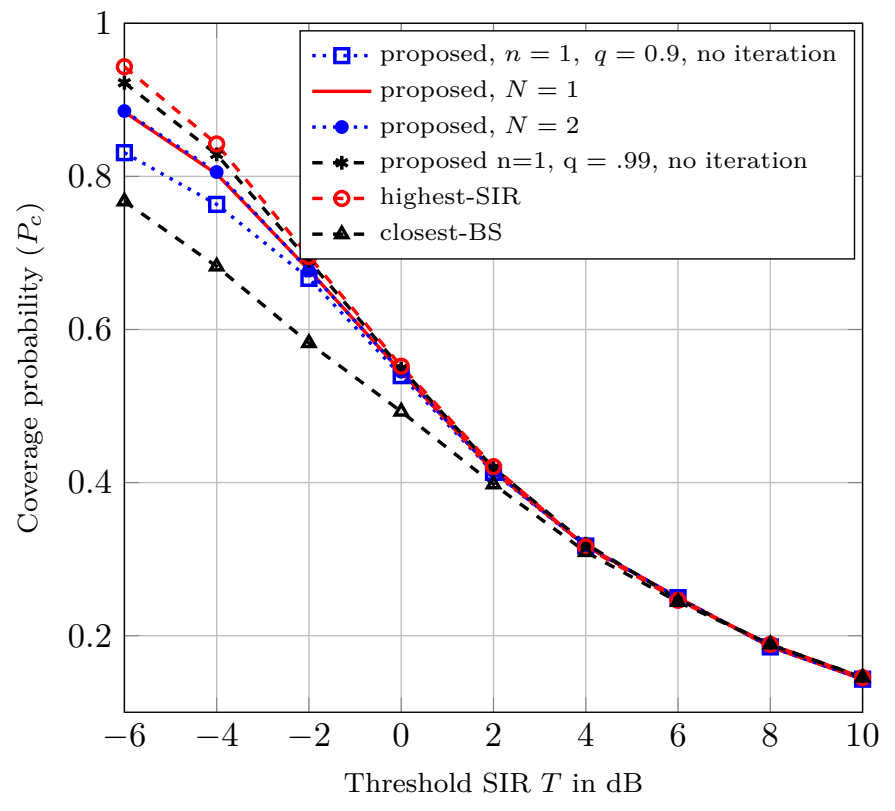

Fig. 3. $P_{c}$ in a single-tier network with adaptive $P_{t h}$ vs $T . \alpha=3.5$, $\lambda=12 \times 10^{-6} \mathrm{~m}^{-2}, P_{t}=20 \mathrm{~W}$.

no iteration outperforms the closest-BS policy. Also, it shows that the performance of the proposed policy with one iteration $(N=1)$ significantly improves $P_{c}$ compared to no iterations. Further, the results indicate that only a marginal performance improvement can be achieved by having more than one iteration. In a network with PPP distribution of BSs, the average number of BSs to be scanned with $N=1$ is given as $q \pi \lambda\left(\frac{P_{t}}{P_{t h}^{(0)}}\right)^{\frac{2}{\alpha}}+$ $(1-q) \pi \lambda\left[\left(\frac{P_{t}}{P_{t h}^{(1)}}\right)^{\frac{2}{\alpha}}-\left(\frac{P_{t}}{P_{t h}^{(0)}}\right)^{\frac{2}{\alpha}}\right]$. For the networks con-

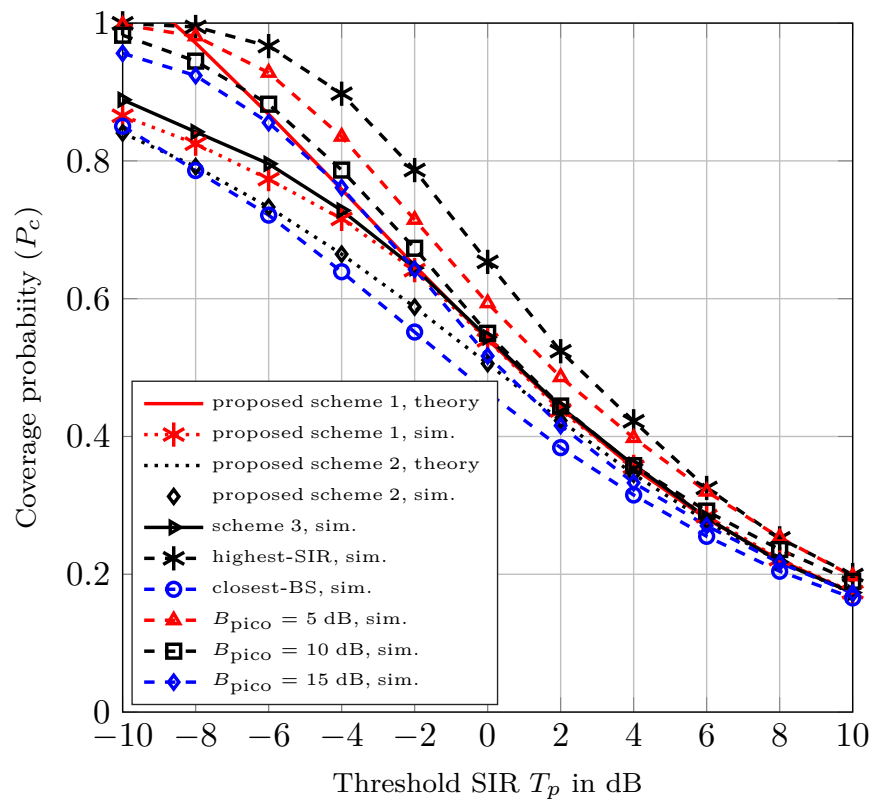

Fig. 4. Variation of $P_{c}$ in a two-tier network with $T_{p} . P_{m}=20 \mathrm{~W}$, $P_{p}=2 \mathrm{~W}, \alpha_{m}=3.5, \alpha_{p}=3.8, n=1, q=0.7, T_{m}=T_{p}-5 \mathrm{~dB}$, $\lambda_{m}=5 \times 10^{-7} \mathrm{~m}^{-2}, \lambda_{p}=20 \times 10^{-6} \mathrm{~m}^{-2}$. Please see footnote ${ }^{3}$ for the description of schemes 1-3 in the legend.

sidered, this figure is $\approx 2.3$. Fig. 3 also shows that $P_{c}$ for $n=1, q=0.99$ no iteration case is only slightly higher compared to $N=1$ case. However, the average number of BSs to be scanned in $n=1, q=0.99$ no iteration case is $\pi \lambda\left(\frac{P_{t}}{P_{t h}}\right)^{\frac{2}{\alpha}} \approx 4.6$. This shows that the complexity (the number of BSs to be tracked) in the association process can be significantly reduced by using adaptive $P_{t h}$ selection at a cost of small performance degradation.

Fig. 4 shows the variation of $P_{c}$ in a two-tier network with the SIR threshold. The minimum average received power $P_{t h}$ for pico-BSs was selected with $n=1$ and $q=0.7$. Under this configuration $70 \%$ MSs will be served by pico-BSs, while the remaining $30 \%$ will be served by macro-BSs. Notably, a considerable coverage probability improvement can be achieved by the availability of both instantaneous SIR and average received power (proposed scheme 1) as opposed to only average received power of pico-BSs (proposed scheme 2), especially when $-8 \mathrm{~dB}$ $<T_{p}<0$ dB. Fig. 4 also shows that having instantaneous SIR information for macro BSs (scheme 3), marginally improves the coverage probability at low SIR thresholds.

${ }^{3}$ Proposed scheme 1: MS is associated with the pico-BS, from which it receives the highest instantaneous SIR, if the average received power from at least this pico-BS exceeds $P_{t h}$; otherwise, the MS is associated with the macro-BS, from which it receives the highest average SIR. Proposed scheme 2: MS is associated with the pico-BS, from which it receives the highest area-averaged received power, if the average received power from at least this pico-BS exceeds $P_{t h}$; otherwise, the MS is associated with the macro-BS, from which it receives the highest average SIR. Scheme 3: MS is associated with the pico-BS, from which it receives the highest instantaneous $\mathrm{SIR}$, if the average received power from at least this pico-BS exceeds $P_{t h}$; otherwise, the MS is associated with the macro-BS, from which it receives the highest instantaneous SIR. 
However, at high SIR thresholds, it does not provide any coverage probability improvement compared to having average SIR. Further, Fig. 4 compares the coverage probabilities of the proposed policy with those of closest-BS, highest-SIR, and maximum biased instantaneous received power [10] association policies. With the closest-BS policy, an MS connects to the closest-BS, while under the highestSIR policy an MS associates with the BS providing the highest SIR, regardless of the BS type. Therefore, these two policies fail to manage user off-loading. Clearly, the highest-SIR policy provides the best coverage probability, while the closest-BS policy provides the worst coverage probability. In maximum biased instantaneous received power [10] association, an MS associates with the macroBS providing the highest instantaneous received power, if the received power from it exceeds the maximum instantaneous received power from pico-BSs with a margin of $B_{\text {pico }}$ $\mathrm{dB}$. Therefore, $B_{\text {pico }}=0 \mathrm{~dB}$ represents the highest SIR association. When $B_{\text {pico }}$ increases, more MSs will be served by pico-BSs than in highest SIR association. Clearly, when $B_{\text {pico }}$ increases, coverage probability decreases due to interference from high power macro-BSs. Results also show that at lower average SIR thresholds, biased association provides better coverage for all $B_{\text {pico values considered }}$ compared to the proposed scheme 1. However, for lower SIR thresholds, the coverage probability of biased association can be lower than that of the proposed scheme 1 depending on the bias $B_{\text {pico }}$.

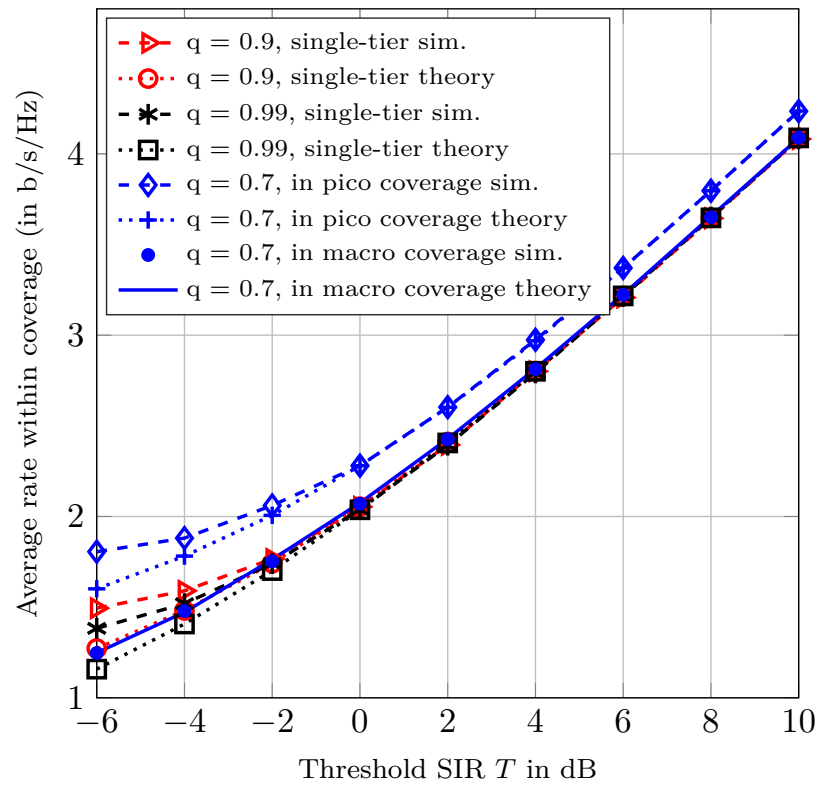

Fig. 5. Average rate of an MS in coverage in a single-tier network, and in the coverage of pico- and macro-tiers in a two-tier network. Network configuration for single tier network: $\alpha=3.5, \lambda=12 \times 10^{-6}$ $\mathrm{m}^{-2}, n=1, P_{t}=20 \mathrm{~W}$. Network configuration for two-tier network: $P_{m}=20 \mathrm{~W}, P_{p}=2 \mathrm{~W}, \alpha_{m}=3.5, \alpha_{p}=3.8, n=1, q=0.7$, $T_{m}=T_{p}=T \mathrm{~dB}, \lambda_{m}=5 \times 10^{-7} \mathrm{~m}^{-2}, \lambda_{p}=20 \times 10^{-6} \mathrm{~m}^{-2}$.

Fig. 5 shows the variation of the average rate of an MS in a single-tier network for two different values of $q$. A close match between theoretical and simulation results can be observed for $T>0 \mathrm{~dB}$. When $T<0 \mathrm{~dB}$, analytical expressions provide lower bounds. Clearly, at high SIR thresholds, MSs in coverage achieve similar average data rates regardless of the value of $q$. However, at low SIR thresholds, average rates slightly decrease when $P_{t h}$ decreases (or equivalently when $q$ and/or $n$ increase). This is because selecting a small $P_{t h}$ allows MSs to associate with BSs located far from them. Fig. 5 also shows the average rate an MS can achieve when in coverage of macro- and pico-BSs assuming cell association described in Section $\mathrm{V}-\mathrm{A}$.

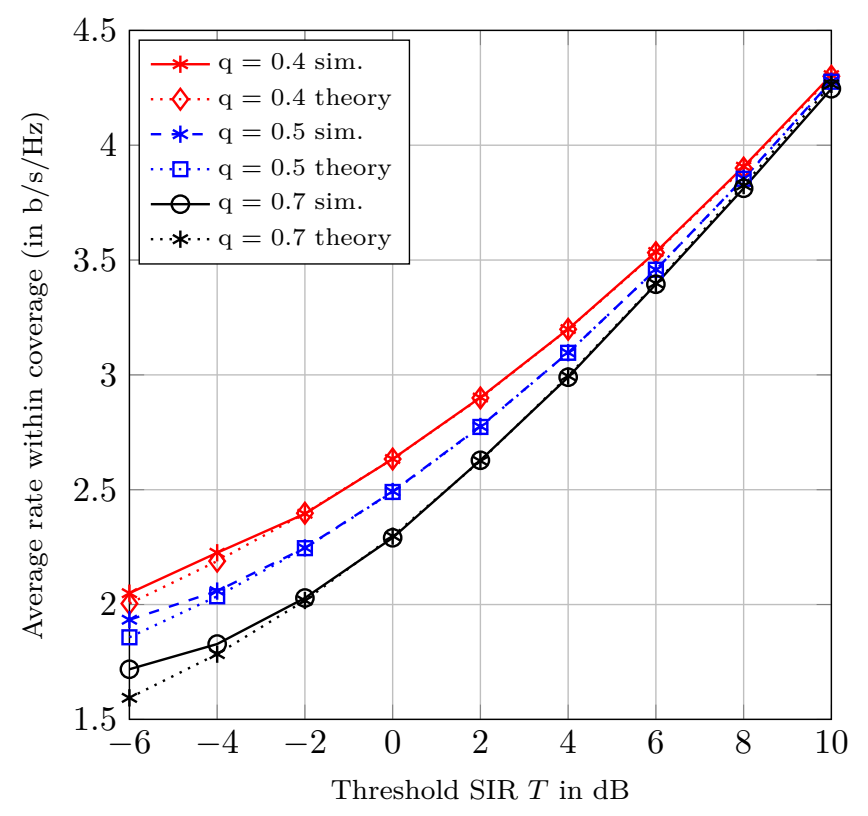

Fig. 6. Average rate of an MS in coverage in a two-tier network for different value of $q . P_{m}=20 \mathrm{~W}, P_{p}=2 \mathrm{~W}, \alpha_{m}=3.5, \alpha_{p}=3.8$, $n=1, T_{m}=T_{p}=T \mathrm{~dB}, \lambda_{m}=5 \times 10^{-7} \mathrm{~m}^{-2}, \lambda_{p}=20 \times 10^{-6} \mathrm{~m}^{-2}$.

The variation of average rates of MSs within coverage in two-tier networks is shown in Fig. 6. Cell association described in Section V-A is assumed. The analytical results closely match the simulation ones. Interestingly, the results show that the average rate increases when $P_{t h}$ for pico-BSs increases. When $P_{t h}$ becomes larger, an MS only associates with a pico-BS that provides higher average SIR (equivalently, with a pico-BS located closer to it). Otherwise, it associates with a macro-BS. Therefore, the effect of strong interference from high power macro-BSs, when an MS is associated with a pico-BS, is limited and average rate increases. However, when $P_{t h}$ is higher, a large percentage of MSs will be associated with macro-BSs, thus reducing the number of users off-loaded to pico-BSs.

\section{CONCLUSION}

Smart cell association policies are critical for emerging heterogeneous cellular networks. This paper has proposed a new cell association policy in which the serving base station (BS) is the one that provides the highest instantaneous SIR from those providing a predetermined minimum average received signal power. This policy includes the conventional highest-SIR association as a special case. Two 
methods to determine the minimum association average received power are given. Application of this policy in both single-tier and two-tier networks are investigated. It has been shown that, this policy outperforms the closestBS policy and performs similarly to the highest-SIR association. In two-tier networks, it can be used to manage traffic off-loading to small cells. Coverage probability and average rate of MSs within coverage of 2- and 3dimensional single-tier (homogeneous) and 2-dimensional two-tier (heterogeneous) networks have been derived and validated by Monte-Carlo simulations. Future research directions may include investigation of the proposed cell association policy in composite fading and when line-ofsight is present, extending it to enable association with more than one BS jointly serving an MS in a coordinated fashion, and investigating the effect of BSs loading on the average achievable rate and coverage.

\section{REFERENCES}

[1] "Cisco visual networking index: Global mobile data traffic forecast update, 2014-2019." Tech. Rep., 2015. [Online]. Available: http://www.cisco.com/c/en/us/solutions/collateral/serviceprovider/visual-networking-index-vni/white_paper_c11520862.pdf

[2] J. Andrews, "Seven ways that HetNets are a cellular paradigm shift," IEEE Commun. Mag., vol. 51, no. 3, pp. 136-144, Mar. 2013.

[3] H. Dhillon, R. Ganti, F. Baccelli, and J. Andrews, "Modeling and analysis of K-tier downlink heterogeneous cellular networks," IEEE J. Sel. Areas Commun., vol. 30, no. 3, pp. 550560, Apr. 2012.

[4] T. Novlan, H. Dhillon, and J. Andrews, "Analytical modeling of uplink cellular networks," IEEE Trans. Wireless Commun., vol. 12, no. 6, pp. 2669-2679, Jun. 2013.

[5] S. Mukherjee, "Distribution of downlink SINR in heterogeneous cellular networks," IEEE J. Sel. Areas Commun., vol. 30, no. 3, pp. 575-585, Apr. 2012.

[6] J. Andrews, F. Baccelli, and R. Ganti, "A tractable approach to coverage and rate in cellular networks," IEEE Trans. Commun., vol. 59, no. 11, pp. 3122-3134, Nov. 2011.

[7] P. Madhusudhanan, J. Restrepo, Y. Liu, T. Brown, and K. Baker, "Stochastic ordering based carrier-to-interference ratio analysis for the shotgun cellular systems," IEEE Wireless Commun. Lett.", vol. 1, no. 6, pp. 565-568, Dec. 2012.

[8] H. Dhillon, R. Ganti, and J. Andrews, "Load-aware heterogeneous cellular networks: Modeling and SIR distribution," in Proc. IEEE Global Communications Conference (GLOBECOM 2012), Dec. 2012, pp. 4314-4319.

[9] P. Madhusudhanan, J. Restrepo, Y. Liu, T. Brown, and K. Baker, "Multi-tier network performance analysis using a shotgun cellular system," in Proc. IEEE Global Communications Conference (GLOBECOM 2011), Dec. 2011, pp. 1-6.

[10] P. Madhusudhanan, J. Restrepo, Y. Liu, and T. Brown, "Downlink coverage analysis in a heterogeneous cellular network," in Proc. IEEE Global Communications Conference (GLOBECOM 2012), Dec. 2012, pp. 4170-4175.

[11] H.-S. Jo, Y. J. Sang, P. Xia, and J. Andrews, "Heterogeneous cellular networks with flexible cell association: A comprehensive downlink SINR analysis," IEEE Trans. Wireless Commun., vol. 11, no. 10, pp. 3484-3495, Oct. 2012.

[12] S. Mukherjee, "Downlink SINR distribution in a heterogeneous cellular wireless network with biased cell association," in Proc. IEEE International Conference on Communications (ICC 2012), Jun. 2012, pp. 6780-6786.

[13] H. Dhillon, R. Ganti, and J. Andrews, "Modeling non-uniform UE distributions in downlink cellular networks," IEEE Wireless Commun. Lett., vol. 2, no. 3, pp. 339-342, Jun. 2013.

[14] S. N. Chiu, D. Stoyan, W. Kendall, and J. Mecke, Stochastic Geometry and Its Applications, 3rd ed. Chichester, UK: John Wiley and Sons, 2013.
[15] M. Haenggi, Stochastic Geometry for Wireless Networks. New York, NY: Cambridge University Press, 2013.

[16] M. Haenggi, J. Andrews, F. Baccelli, O. Dousse, and M. Franceschetti, "Stochastic geometry and random graphs for the analysis and design of wireless networks," IEEE J. Sel. Areas Commun., vol. 27, no. 7, pp. 1029-1046, Sep. 2009.

[17] H. Wang and M. Reed, "A novel tractable framework to analyse heterogeneous cellular networks," in Proc. 6th IEEE Joint Workshop on Heterogeneous, Multi-Hop, Wireless and Mobile Networks, co-located with IEEE GLOBECOM 2011, Dec. 2011, pp. 287-292.

[18] _ , "Tractable model for heterogeneous cellular networks with directional antennas," in Proc. Australian Communications Theory Workshop (AusCTW 2012), Jan.-Feb. 2012, pp. 61-65.

[19] P. Herath, W. Krzymien, and C. Tellambura, "A novel base stations - mobile stations association policy for cellular networks," in Proc. 80th IEEE Vehicular Technology Conference (VTC 2014-Fall), Vancouver, Canada, Sep. 2014.

[20] I. S. Gradshteyn and I. M. Ryzhik, Table of Integrals, Series and Products, 7th ed. San Diego, CA: Academic Press, 2007.

[21] J. F. C. Kingman, Poisson Processes. New York, NY: Oxford University Press, 1993.

[22] M. Haenggi, "On distances in uniformly random networks," IEEE Trans. Inf. Theory, vol. 51, no. 10, pp. 3584-3586, Oct. 2005 . 